\title{
Hypothesis Generation for a Possible Mechanism of Transmural Reentry Following a Histological Appraisal of Postmortem Atria
}

\author{
Ciara Mahon, MSc, MRCP ${ }^{1, *}$, Louisa $O^{\prime} N e i l l, M R C P^{1}$, Mark Henesy, MRCP ${ }^{1}$, Sara Kelly, MSc ${ }^{2}$, Aurelie \\ Fabre, PhD, FRCPath, FRCPI' and David Keane, PhD, FHRS, FRCPI \\ ${ }^{1}$ Department of Cardiology, St Vincent's University Hospital, Ireland \\ ${ }^{2}$ Department of Histopathology, St Vincent's University Hospital, Ireland
}

\begin{abstract}
Objective: To provide support for the existence of epicardial to endocardial connection following a histological appraisal of post-mortem atria.

Background: The relationship between atrial fibrillation $(\mathrm{AF})$ and atrial anatomy is well recognized and myocardial fibre orientation is known to influence conduction velocity. The precise mechanism of AF perpetuation remains elusive. The prevailing but contrasting hypotheses are re-entrant mechanisms and focal mechanisms. Support for such a unifying mechanism could be provided by finding an anatomical substrate to support epicardial to endocardial connections.

Methods: A prospective single centre post-mortem appraisal. Histological atrial samples were systematically obtained from pre-specified areas in ten cadaveric hearts. A pre-morbid history of both AF and non-AF were included. Samples were fixed and stained. Microscopic histological analysis was performed.

Results: Previously reported and well recognised myocardial orientation patterns were noted. In addition, myocardial fibres connecting the epicardial and endocardial surface were seen, and an apparent separation zone containing fibrosis and adipocytes within the atria.

Conclusion: To our knowledge this is the first study that provides histological support for myocardial fibres connecting the epicardial to endocardial surface. This was a pilot study and there were no observable histological differences between atrial tissue obtained from patients with AF and without AF. This finding may be non-specific. However, it does expand on previous documented anatomical structure. Further allows for hypothesis generation in the possibility of this myocardial fibre orientation involvement in epicardial to endocardial breakthrough. Further investigations would be required for more definitive results.
\end{abstract}

\section{Keywords}

Epicardial breakthrough, Myocardial orientation, Left atrium, Atrial fibrillation, Post-mortem study, Histological, Reentrant mechanisms, Focal mechanisms

\section{Introduction}

Atrial Fibrillation (AF) is the most common sustained arrhythmia and is associated with increased cardiovascular morbidity and mortality [1]. The relationship between AF and atrial anatomy is well recognized and myocardial fibre orientation is known to influence conduction velocity. However, the precise mechanism of AF perpetuation remains elusive. This gap in knowledge leads to a paucity in treatment development. Two of the most prevailing but contrasting hypotheses in the field of AF are re-entrant mechanisms (including multiple wavelets and the existence of re-entry circuits) and focal mechanisms (including repetitive spontaneous focal discharges) [2-4]. A third potentially unifying hypothesis has been proposed termed the double layer hypothesis. The double layer hypothesis proposes that apparent focal discharges on the epicardium may reflect breakthrough from re-entrant

*Corresponding author: Ciara Mahon, MSc, MRCP, Email: c.mahon@rbht.nhs.uk

Accepted: March 14, 2020

Published online: March 16, 2020

Citation: Mahon C, O'Neill L, Henesy M, et al. (2020) Hypothesis Generation for a Possible Mechanism of Transmural Reentry Following a Histological Appraisal of Postmortem Atria. J Cardiothorac Surg Ther 4(1):46-51 
Citation: Mahon C, O'Neill L, Henesy M, et al. (2020) Hypothesis Generation for a Possible Mechanism of Transmural Reentry Following a Histological Appraisal of Postmortem Atria. J Cardiothorac Surg Ther 4(1):46-51

activity on the endocardium rather than spontaneous ectopy $[5,6]$.

The evidence supporting either of the two former hypotheses arises from endocardial and epicardial mapping studies in animal and human hearts and the relationship to the density of contact mapping electrodes $[3,4]$. Clinical studies have identified focal and re-entrant AF drivers in both the right and left human atria using epicardial or endocardial mapping techniques [7-9]. More recently magnetic resonance three dimensional structural-functional mapping of diseased human right atria ex-vivo studies revealed that complex atrial microstructure caused significant differences between endocardial versus epicardial activation during pacing and sustained atrial fibrillation [10].

Our study hypothesis was aimed at providing evidence for existence of epicardial to endocardial connection following a histological appraisal of post-mortem atria. That would add to volume of literature on the mechanism of $A F$, and perhaps a substrate for further investigation.

\section{Methods}

\section{Post-mortem}

Ethical approval was obtained from the hospital research ethics committee as per the Declaration of Helsinki for Ethical Principles for Medical Research Involving Human Subjects and conducted in full conformity with relevant regulations and with the ICH Guidelines for Good Clinical Practice (CPMP/ICH/135/95) July 1996. Informed verbal consent was obtained prior to the autopsy, printed study detail was forwarded to the next of kin and written consent obtained prior to processing and analysis of the biopsies.

Ten hearts were explanted from deceased patients for whom an autopsy was performed to establish the cause of death. The hospital autopsy protocol was followed in all cases and a specialist cardiac histopathologist obtained the biopsies.

\section{Atrial tissue sampling}

Myocardial orientation pattern vary throughout the atrium, therefore samples were systematically taken from 10 pre-specified areas in all hearts, 1) The posterior right atrium; 2) The inter-atrial septum near pulmonary veins; 3 ) The inter-atrial septum near Bachmann's Bundle [11-13]; 4) The coronary sinus; 5) The cavo-tricuspid isthmus; 6) The apical left atrial appendage; 7) The mid left atrial appendage; 8) The basal left atrial appendage; 9) The right atrium appendage and 10) The posterior left atrium near right upper pulmonary vein. Each specimen included epicardial and endocardial/wall to wall at the time of autopsy.

\section{Histological analysis}

After fixation in $4 \%$ neutral buffered formalin the atrial tissue were embedded in paraffin, sectioned and stained with hematoxylin and eosin (H\&E) stain and elastic stain.

The predominant myocardial fibre orientation was inde- pendently determined by two investigators under the direction of a cardiac histopathologist. Any discrepancy was re-assessed and adjudicated by a cardiac histopathologist.

\section{Statistical analysis}

A chi-squared test was used to determine if any significant difference between orientation patterns in AF patients compared to non-AF patients.

\section{Results}

\section{Post-mortem}

Ten explanted hearts were examined. In all cases the cause of death was unrelated to cardiac disease. Five men and five women aged $74.4 \pm 13.1$ years (mean \pm SD) were included. Four patients had previously documented AF, and six patients did not have documented AF.

\section{Macroscopic}

The complex 3-dimensional atrial musculature was consistent with previous anatomical observations [14,15]. The right atrium was dominated by arrays of pectinate muscle within an extensive appendage while the left atrium was relatively smooth walled, with a small tubular appendage.

\section{Total slides analysed by microscopy}

Two sections were examined from each area biopsied within the heart. A total of 190 slides were included. Seventy slides were obtained from AF patients, and 120 slides were obtained from non-AF patients. Ten slides were not analysed because of technical quality during the embedding stage.

\section{Slides with an epicardial to endocardial/wall to wall identified}

Both an epicardial and endocardial border was identified on $74.2 \%$ ( $n=141$ of 190$)$ slides. Of these slides $77 \%$ ( $n=54$ of 70 ) were samples from AF patients, and $72.5 \%(n=87$ of 120$)$ were samples from non-AF patients.

\section{Myocardial fibre pattern}

Slides were further analysed for myocardial fibre pattern which we termed 1) Fibres connecting the epicardial to endocardial surface; 2) Well-organized fibre; 3) Disorganized fibres "serpiginous-like" and 4) An apparent separation (see Figure 1 and Figure 2).

1. A fibre connecting the epicardial to endocardial border was identified in $24.1 \%$ ( $n=34$ of 141 ) of samples. In AF patients this represented $22.2 \%$ ( $n=12$ of 54 ) of these slides, and in non-AF patients $25.2 \%$ ( $n=22$ of 87 ).

2. A well-organized myocardial fibre pattern was identified in $15.8 \%$ ( $n=30$ of 190 ) of samples. In AF patients this represented $18.5 \%$ ( $n=13$ of 70 ) of these slides, and in non-AF patients $14.1 \%$ ( $n=17$ of 120 ) of these slides.

3. A disorganized "serpiginous" fibre pattern was identified in $40.5 \%$ ( $n=77$ of 190) of samples. In AF patients this represented $41.4 \%$ ( $n=29$ of 70 ) of these slides, and in non-AF patients $40 \%$ ( $n=48$ of 120 ) of these slides. 
Citation: Mahon C, O'Neill L, Henesy M, et al. (2020) Hypothesis Generation for a Possible Mechanism of Transmural Reentry Following a Histological Appraisal of Postmortem Atria. J Cardiothorac Surg Ther 4(1):46-51

A.

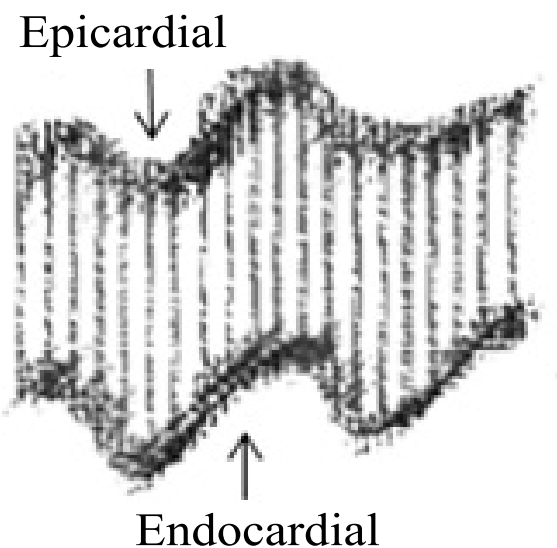

B.

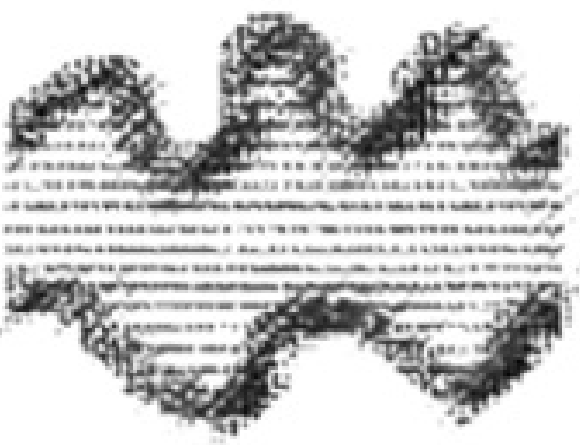

C.

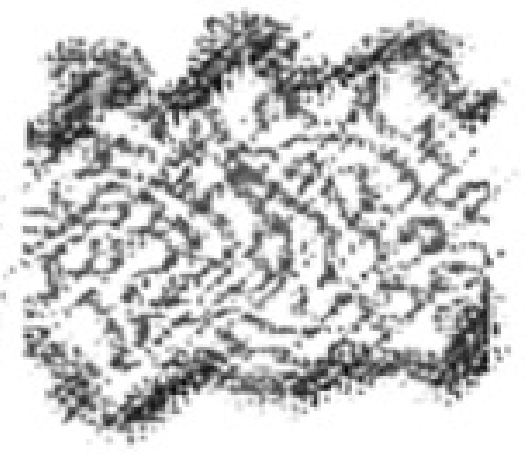

Figure 1a: Schematic representation: To depict classification of myocardial fiber orientation. A) Myocardial bundles connecting the endocardial to the epicardial surface; B) Well organized myocardial bundles not connecting the epicardial and endocardial surface. C) Serpiginous myocardial bundles in a whorled/less organized pattern.
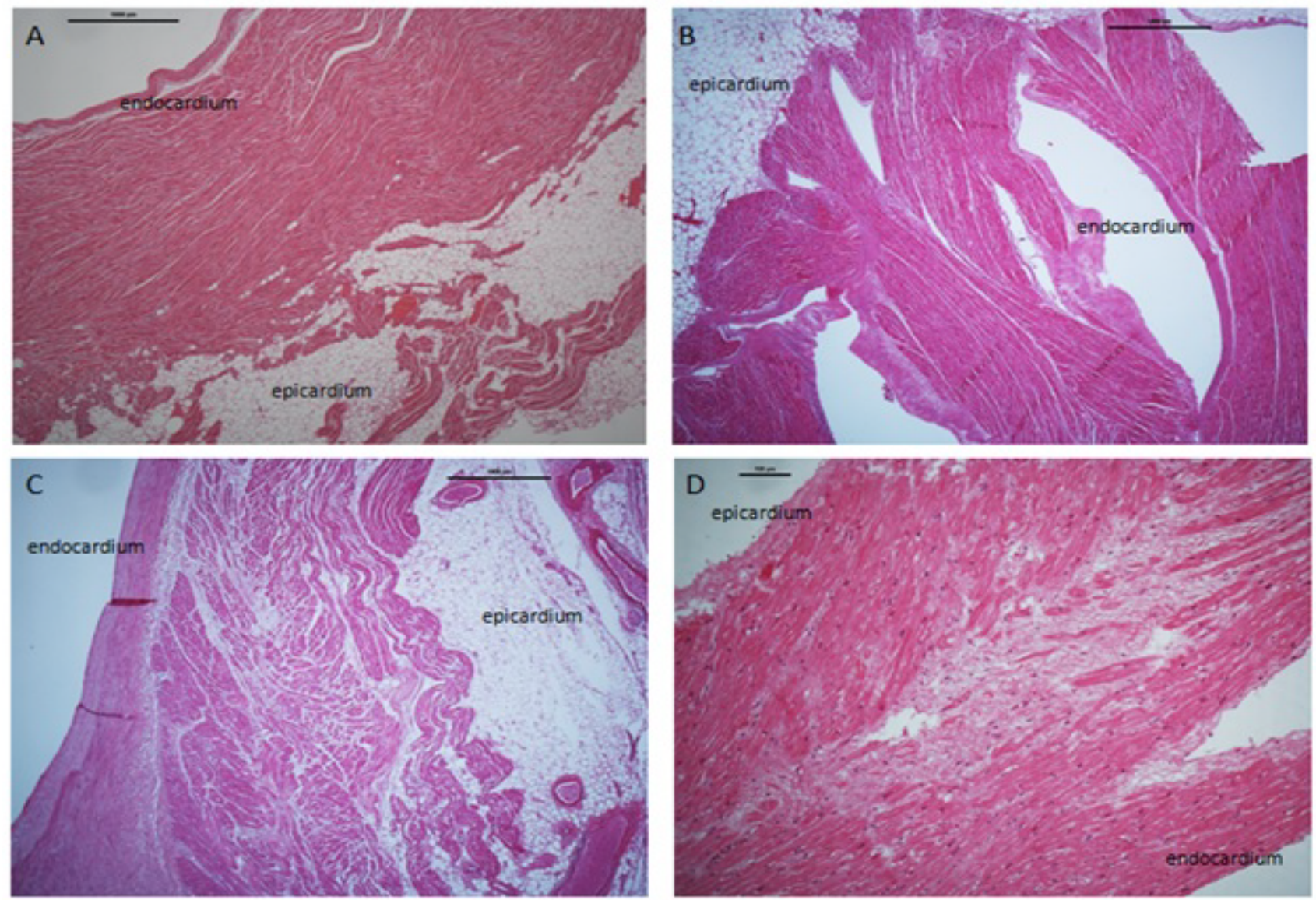

Figure 1b: A) Represents well organized myocardial bundles relative to the endocardial and epicardial border from the cavo-tricuspid isthmus; B) Represents myocardial fibres connecting the endocardial and epicardial border from the apical left atrial appendage; C) Represents serpiginous myocardial orientation relative to the endocardial and epicardial border from the posterior wall of the posterior wall of the left atrium; D) Separation of the sub-epicardial and sub-endocardial border from the mid posterior right atrium.

4. An apparent separation zone between the epicardial and endocardial layers was identified in $8.9 \%$ ( $n=17$ of 190) of all slides. In AF patients this represented $12.9 \%(n=9$ of 70 ), and in non-AF patients $6.6 \%$ ( $n=8$ of 120 ) of slides.
The separation zone contained adipocytes and fibrous tissue.

There was no significant difference in myocardial fibre pattern found between AF patients versus non-AF patients 
Citation: Mahon C, O'Neill L, Henesy M, et al. (2020) Hypothesis Generation for a Possible Mechanism of Transmural Reentry Following a Histological Appraisal of Postmortem Atria. J Cardiothorac Surg Ther 4(1):46-51

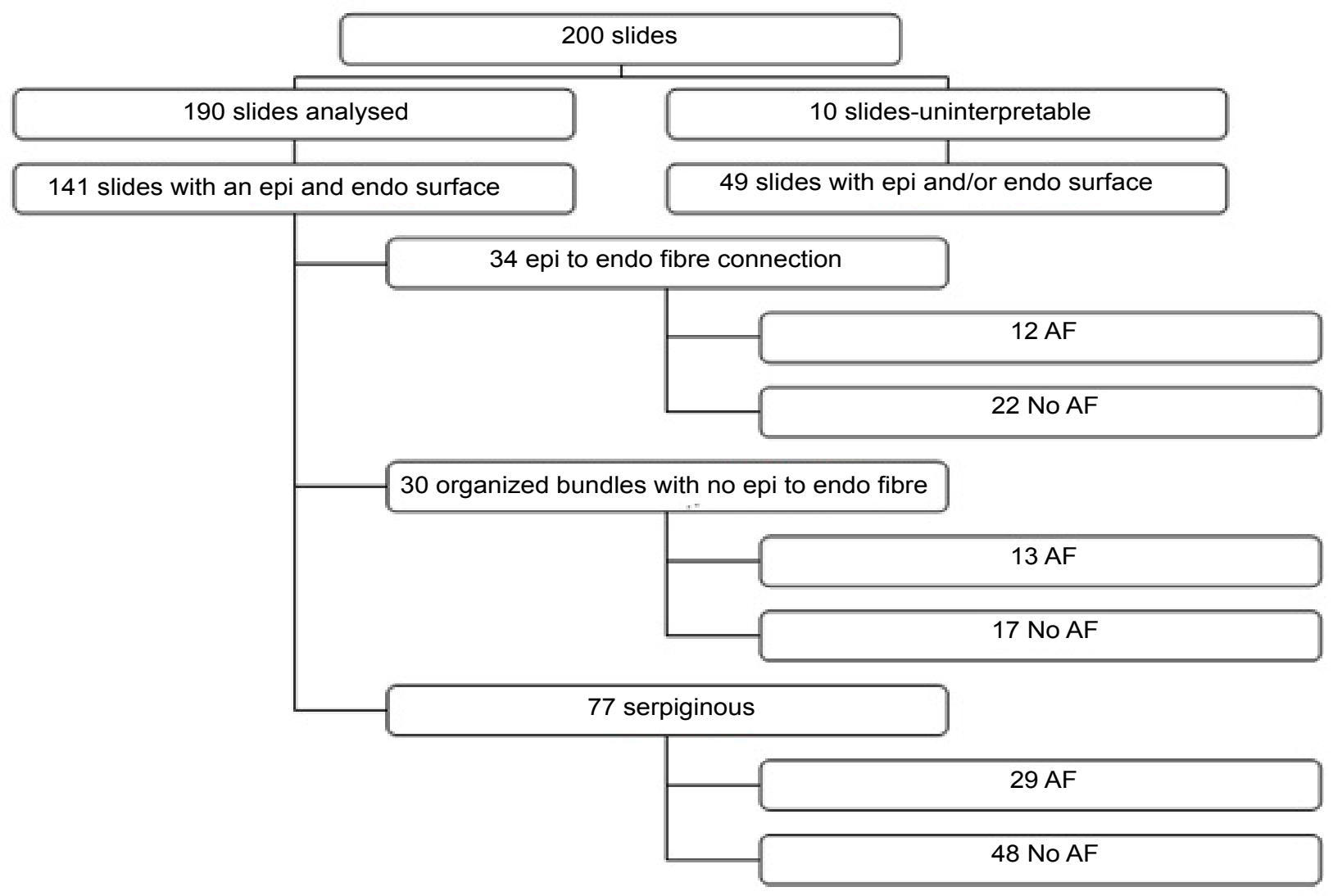

Figure 2: Slide interpretation: The number of slides interpreted, and the subsequent predominant myocardial orientation patterns found.

AF: Atrial Fibrillation; SR: Sinus Rhythm; Epi: Epicardial and Endo: Endocardial.

using the chi square test. The chi-square test was 0.465 . The p-value was not significant at 0.79 .

\section{Discussion}

The aim of this post-mortem study was to highlight a novel atrial finding that adds to the growing volume of literature on the mechanisms of atrial fibrillation. The concept that myocardial structure affects AF propagation is well accepted. Reduced action potential duration and tissue fibrosis are thought to be important in promoting re-entry and maintain AF [16-19]. Focal firing occurs mainly in the architecture of pulmonary veins, via enhanced automaticity or triggered activity because of early or delayed after depolarisations $[16,17,20,21]$. Local ectopic activity can act as an AF-maintaining ectopic driver or can trigger AF-maintaining re-entry in a vulnerable myocardial substrate. Voigt, et al. described how the complex changes in cellular and molecular electrophysiology contribute to the occurrence of focal firing via ion current and calcium handling abnormalities to produce a re-entrant substrate via electric remodelling. They further described a relationship between cellular and molecular electrophysiological determinants in the progression of structural remodelling [21]. However, the proposed relationship between myocardial fibre organization and patterns of re-entry in fibrillation is not sufficiently developed considering the existing background literature on the topic. With evolving interest in the conceptual models of $\mathrm{AF}$, including re-entrant and focal mechanisms, there has been a renewed interest in understanding the gross and microscopic arrangements of atrial musculature to provide further morphological basis for atrial conduction. This study provides a unique description of myocardial fibres connecting the epicardial to the endocardial surface. This further expands on previous studies, and we propose a potential link to this myocardial fibre orientation and the mechanism of AF.

Atrial myoarchitecture has been well described [12,14]. Consistent with previous studies our histological study found well organised myocardial fibres in addition to myocyte fibres that appear to connect the epicardial and endocardial surface in human atria. To our knowledge this bridging of individual myocyte fibres between epicardial and endocardial noted in this study has not been previously described. Our study did not find any statistical significance in the number of discrete foci between AF and non-AF patients. However, this study was aimed at identifying unique myocardial features. Further studies with larger sample size in patients with AF and nonAF patients would be required to determine if any statistical significance exists.

The role of myocardial fibres running between the endocaridal and epicaridal surface could be non-specific. However, one potential role for these epicardial and endocardial fibres could be to facilitate propagation, a conduction substrate, for "epicardial breakthrough". De Groot, et al. 
Citation: Mahon C, O'Neill L, Henesy M, et al. (2020) Hypothesis Generation for a Possible Mechanism of Transmural Reentry Following a Histological Appraisal of Postmortem Atria. J Cardiothorac Surg Ther 4(1):46-51

provided the first evidence for asynchronous activation of the endo-epicardial wall during AF in humans, and suggested that endo-epicardial asynchrony may play a major role in the pathophysiology of AF [22]. Overall, he explains that the presence of focal fibrillation waves during AF can be explained by asynchronous activation of the atrial endo- and epicardial layer and transmurally propagating fibrillation waves.

Secondly, the impediment to study that relationship of myocardial orientation patterns stems from technological challenges in mapping propagation, mainly inside the thick myocardium, and less in challenges of myocardial organization descriptions. Trayanova reviewed the evolution of mathematical modelling and computer simulation approaches, such as atrial cell action potential models, 2-dimensional (2D) and 3D tissue models, and recent developments in the reproduction of realistic atrial tissue geometries [23].

The proposed relationship between myocardial fibres organization and patterns of re-entry in fibrillation is not sufficiently developed considering the existing background literature on the topic. Re-entrant activity in three-dimensional cardiac muscle may be organized as a scroll wave rotating around a singularity line called a filament $[24,25]$. Mapping of the epicardial surface during ventricular tachycardia or fibrillation has revealed re-entrant patterns of activity that are consistent with transmural scroll waves [26]. Using computer models, theoretically the filament of a 3D rotor/a scroll wave has been predicted to tend to align with fibres [27] and to follow the path of least electrical resistance [28]. One implication of such theoretical predictions is that the filament of intramural scroll waves would follow fibres directions or dissociation between fibre layers either parallel or perpendicular to the epicardial to endocardial layers. Myocardial fibres connecting the epicardial to the endocardial described in this study may provide a substrate for this filament. However, scroll waves are also affected by the boundaries of the myocardium. However, this leads to another implication of the theory that is relevant to AF. AF is propagated in the thin walled atrium rather than the relatively thicker ventricle. The scroll waves should be more stable in the transmural configuration (filament extended between the endocardial to epicardial surface) rather than in the intramural one, regardless of the organization of the fibres.

Our data supports the existence of endo-epicardial connections. The implication of this remains to be proven. However, the advent of new imaging modalities including the application of sub-millimeter diffusion tensor magnetic resonance imaging and 3D computer models have provided further information on both human atrial structure, as well as inter subject variability [29]. A human atrial model with fibre orientation has been used to investigate the relationship between different atrial arrhythmic propagation patterns and the electrograms observed on the atrial surface [30]. High resolution and fidelity of this data could enhance our understanding of atrial structural contributions, including endo-epicardial connections, to atrial rhythm and pump disorders and lead to improvements in their targeted treatment.

A final note on the apparent separation zone between endocardial and epicardial layers. The separation zones contain adipocytes and fibrosis. Studies have revealed localized AF drivers in both the left and right atria [7-9] whereas other groups using epicardial mapping have demonstrated more wave breaks than localized re-entrant activity [4-8]. One study demonstrated that fibrosis can act as an architectural discontinuity between atrial pectinate muscle and small intramural bundles leading to both longitudinal and intramural conduction block, especially during fast atrial pacing [10]. In their study they used simultaneous sub-epicardial and sub-endocardial mapping combined with detailed 3-dimensional atrial microstructure imaging to demonstrate that the complex atrial microstructure caused significant differences between endocardial versus epicardial activation during pacing and sustained AF driven by intramural re-entry anchored to fibrosis-insulated atrial bundles [10]. Our findings of an apparent separation zone between the sub-epicardial and sub-endocardial layers could add to the concept of bilayer re-entry. If the sub-endocardium and sub-epicardium are activated in different directions and are in places separated by an anatomical separation zone then it is difficult to conceive that current body-surface mapping systems could detect such subtleties and be prone to provide inaccurate summation vector cardiograms of the full atrial wall during $A F$ rather than representing actual real-time activity in either the sub-endocardial or the sub-epicardial layers.

Overall, this is a novel study looking in to the microscopic fibre orientation in AF and non-AF patients. The study supports evidence of epicardial to endocardial fibre connections, albeit the implications of this finding need further investigation.

\section{Conclusion}

To our knowledge this is the first study that provides histological support for myocardial fibres connecting the epicardial to endocardial surface. This was a pilot study and there were no observable histological differences between atrial tissue obtained from $A F$ and without AF. This finding may be non-specific. However, it does expand on previous documented anatomical structure and raises the possibility for involvement in epicardial to endocardial breakthrough. Further investigations would be required for more definitive results.

\section{Conflict of Interest}

There are no conflicts of interest.

\section{Funding}

Irish Heart Foundation. Bursary award number 1199348.

\section{Ethical Approval}

The research reported in this paper adhered to St. Vincent's University Hospital, Ireland, Ethical committee guidelines, and Guideline on Good Clinical Practice (ICH topic E6, step 4, CPM/ICH 135/95).

\section{Background Notes}

In experimental animal models high-resolution optical mapping demonstrate that pacing-induced $A F$ is due to the 
Citation: Mahon C, O'Neill L, Henesy M, et al. (2020) Hypothesis Generation for a Possible Mechanism of Transmural Reentry Following a Histological Appraisal of Postmortem Atria. J Cardiothorac Surg Ther 4(1):46-51

presence of a very rapid rotor in the left atrium (6 and 7). However, whether this mechanism occurs in true in longstanding persistent AF remains to be clarified (6 and 7). Following a clinical series, in which the electrical activity of the atria was mapped by two basket catheters it was postulated that human $\mathrm{AF}$ is due to the presence of a stable rotor that serves as a driver to sustain AF. Ablation of the centre of these rotors abruptly terminated or consistently slowed $A F$ in majority of cases, and substantially improved long-term freedom from AF compared to conventional ablation alone.

It is unclear whether these two opposing views are mutually exclusive. Potential mechanisms for appearance of focal activation in epicardium could be 3D breakthrough or ectopic firing. Considering ongoing debate, we studied myocardial fiber orientation to determine this could support a substrate for 3D and break though (11).

\section{References}

1. Kunihiro N, Stanley N (2014) Atrial fibrillation compendium: Historical context and detailed translational perspective on an important clinical problem. Circ Res 114: 1447-1452.

2. Allessie M, de Groot N (2014) CrossTalk opposing view: Rotors have not been demonstrated to be the drivers of atrial fibrillation. J Physiol 592: 3167-3170.

3. Mandapati R, Skanes A, Chen J, et al. (2000) Stable microreentrant sources as a mechanism of atrial fibrillation in the isolated sheep heart. Circulation 101: 194-199.

4. Jalife J, Berenfeld O, Mansour M (2002) Mother rotors and fibrillatory conduction: A mechanism of atrial fibrillation. Cardiovasc Res 54: 204-216.

5. de Groot NM, Houben RP, Smeets JL, et al. (2010) Electropathological substrate of longstanding persistent atrial fibrillation in patients with structural heart disease: Epicardial breakthrough. Circulation 122: 1674-1682.

6. Eckstein J, Maesen B, Linz D, et al. (2011) Time course and mechanisms of endo-epicardial electrical dissociation during atrial fibrillation in the goat. Cardiovasc Res 89: 816-824.

7. Shivkumar K, Ellenbogen KA, Hummel JD, et al. (2012) Acute termination of human atrial fibrillation by identification and catheter ablation of localized rotors and sources: First multicenter experience of focal impulse and rotor modulation (FIRM) ablation. J Cardiovasc Electrophysiol 23: 1277-1285.

8. Lee G, Kumar S, Teh A, et al. (2014) Epicardial wave mapping in human long-lasting persistent atrial fibrillation: Transient rotational circuits, complex wavefronts, and disorganized activity. Eur Heart J 35: 86-97.

9. Narayan SM, Krummen DE, Clopton P, et al. (2013) Direct or coincidental elimination of stable rotors or focal sources may explain successful atrial fibrillation ablation: On-treatment analysis of the CONFIRM trial (Conventional ablation for AF with or without focal impulse and rotor modulation). J Am Coll Cardiol 62: 138-147.

10. Hansen BJ, Zhao J, Csepe TA, et al. (2015) Atrial fibrillation driven by micro-anatomic intramural re-entry revealed by simultaneous sub-epicardial and sub-endocardial optical mapping in explanted human hearts. Eur Heart J 36: 2390-2401.

11. van Campenhout M, Yaksh A, Kik C, et al. (2013) Bachmann's bundle. Circ Arrhythmia Electrophysiol 6: 1041-1046.
12. Lemery R, Guiraudon G, Veinot JP (2003) Anatomic description of Bachmann's bundle and its relation to the atrial septum. Am J Cardiol 91: 1482-1485.

13. Ho SY, Sanchez-Quintana D (2009) The importance of atrial structure and fibers. Clin Anat 22: 52-63.

14. Ho SY, Anderson RH, Sánchez-Quintana D (2002) Atrial structure and fibres: Morphologic bases of atrial conduction. Cardiovasc Res 54: 325-336.

15. Loukas M, Tubbs RS, Tongson JM, et al. (2008) The clinical anatomy of the crista terminalis, pectinate muscles and the teniae sagittalis. Ann Anat - Anat Anzeiger 190: 81-87.

16. Nattel S (2002) New ideas about atrial fibrillation 50 years on. Nature 415: 219-226.

17. Yu-ki I, Kunihiro N, Takeshi K, et al. (2011) Atrial fibrillation pathophysiology. Circulation 124: 2264-2274.

18. Lixia Y, Jianlin F, Rania G, et al. (1997) Ionic remodeling underlying action potential changes in a canine model of atrial fibrillation. Circ Res 81: 512-525.

19. Danshi L, Samir F, Ki LT, et al. (1999) Promotion of atrial fibrillation by heart failure in dogs: Atrial remodeling of a different sort. Circulation 100: 87-95.

20. Leif H-M, Anna L, Antoni B-G, et al. (2004) Atrial fibrillation is associated with increased spontaneous calcium release from the sarcoplasmic reticulum in human atrial myocytes. Circulation 110: 1358-1363.

21. Voigt N, Heijman J, Wang Q, et al. (2014) Cellular and molecular mechanisms of atrial arrhythmogenesis in patients with paroxysmal atrial fibrillation. Circulation 129: 145-156.

22. de Groot N, van der Does L, Yaksh A, et al. (2016) Direct proof of endo-epicardial asynchrony of the atrial wall during atrial fibrillation in humans. Circ Arrhythmia Electrophysiol 9: e003648.

23. Trayanova NA (2014) Mathematical approaches to understanding and imaging atrial fibrillation: Significance for mechanisms and management. Circ Res 114: 1516-1531.

24. Medvinsky AB, Panfilov AV, Pertsov AM (1984) Properties of rotating waves in three dimensions. Scroll Rings in Myocard BT - Self-Organization Autowaves and Structures Far from Equilibrium. In: Krinsky VI, Springer Berlin Heidelberg, Heidelberg, Berlin, 195-199.

25. Winfree AT (1994) Electrical turbulence in three-dimensional heart muscle. Science 266: 1003-1006.

26. Gray RiA, Jalife J (1996) Spiral Waves and the Heart. Int J Bifurc Chaos 06: 415-435.

27. Berenfeld O, Pertsov AM (1999) Dynamics of intramural scroll waves in three-dimensional continuous myocardium with rotational anisotropy. J Theor Biol 199: 383-394.

28. Wellner M, Berenfeld O, Jalife J, et al. (2002) Minimal principle for rotor filaments. Proc Natl Acad Sci U S A 99: 8015-8018.

29. Farhad PA, Herzka DA, Hiroshi A, et al. (2016) Myofiber architecture of the human atria as revealed by submillimeter diffusion tensor imaging. Circ Arrhythmia Electrophysiol 9: e004133.

30. Tobón C, Ruiz-Villa CA, Heidenreich E, et al. (2013) A three-dimensional human atrial model with fiber orientation. Electrograms and arrhythmic activation patterns relationship. PLoS One 8: e50883. 